\title{
A chilling link to joint pain and hypersensitivity
}

Two independent studies published in Arthritis Research \& Therapy identified TRPA1 (transient receptor potential cation channel subfamily A member 1), a cold-sensing, calciumpermeable channel expressed in neurons, fibroblasts and cells of the immune system, as a component of mechanonociception and coldinduced hyperalgesia in a mouse model of arthritis.

Previous research into the role of TRPA 1 in the context of inflammation showed that deletion of Trpal or the use of TRPA1 antagonists can ameliorate pain in mice. Zsuzsanna Helyes and colleagues observed milder symptoms of adjuvant-induced arthritis in Trpa1-knockout mice than in wild-type controls, including reduced inflammation, histopathology and nociception. Using a similar mouse model of arthritis, Susan Brain and colleagues observed a
TRPA1-dependent exacerbation of pain behaviour after cold exposure that was associated with increased blood flow to adjuvant-treated joints.

These two studies suggest that TRPA1 mediates nociception in inflammatory arthritis, and that low temperatures might potentiate pain. "We believe that TRPA1 may act locally within the joint to influence blood flow via sensory nerves, and that this could be related to the pain response and its worsening in the cold," explains Brain. "Clinical trials on novel TRPA1 antagonists as analgesics for neuropathic pain are ongoing, but it's too early to say whether TRPA1 antagonists will be effective in [ameliorating]

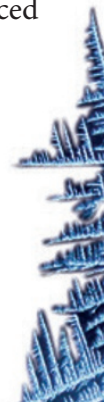

According to Helyes, "the main advantage of TRPA1 as a potential drug target in arthritis and related pain is that TRPA1 is an important sensor molecule both in nociceptors and inflammatory cells, integrating different inflammatory stimuli."

João H. Duarte

ORIGINAL ARTICLES Horváth, Á. et al.

Transient receptor potential ankyrin 1 (TRPA1) receptor is involved in chronic arthritis: in vivo study using TRPA1-deficient mice. Arthritis Res. Ther. 18, 6 (2016) |Fernandes, E. S. et al.

Environmental cold exposure increases blood flow and affects pain sensitivity in the knee joints of CFA-induced arthritic mice in a TRPA1-dependent manner. Arthritis Res. Ther. 18, 7 (2016)

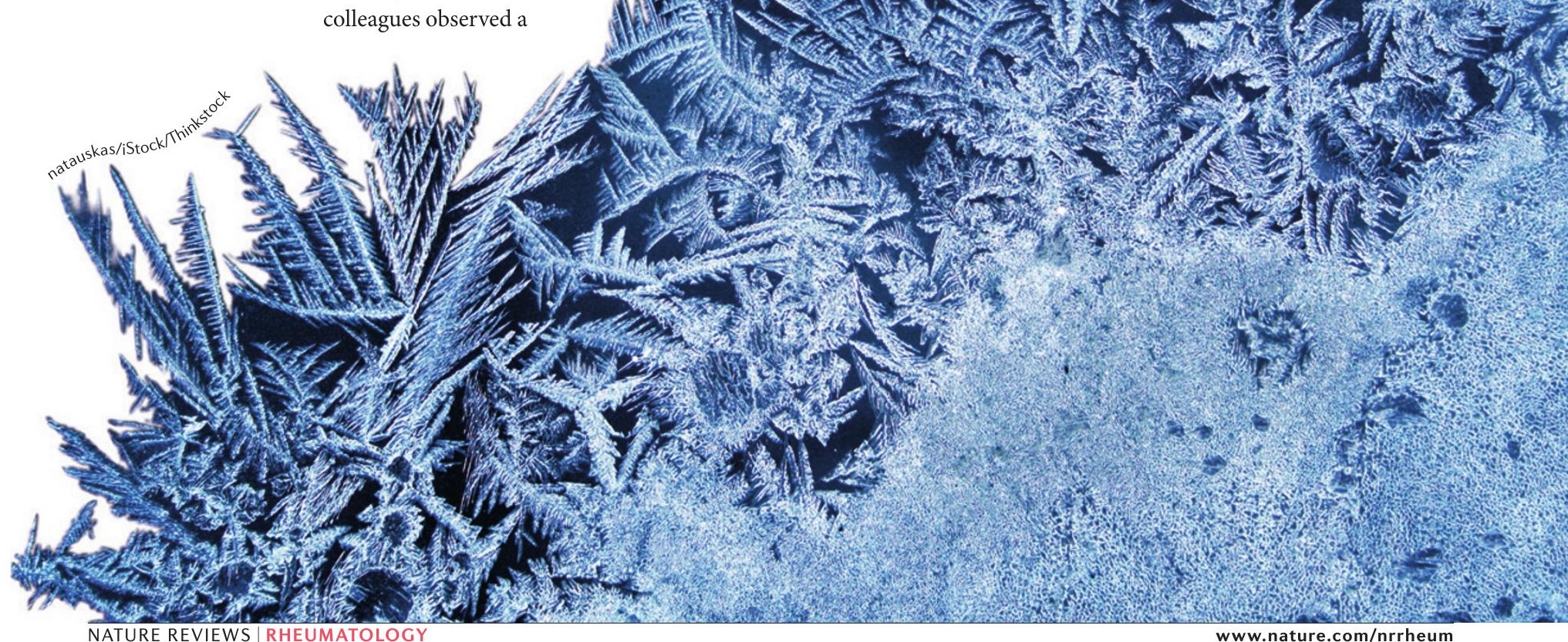

